\title{
The Basic Idea behind the Relativistic and the Quantum Concepts
}

\author{
Ahmed Isam \\ Khartoum, Sudan \\ Email: ahmedisam777@gmail.com
}

Received 20 December 2015; accepted 23 January 2016; published 28 January 2016

Copyright (C) 2016 by author and Scientific Research Publishing Inc.

This work is licensed under the Creative Commons Attribution International License (CC BY). http://creativecommons.org/licenses/by/4.0/

c) (i) Open Access

\begin{abstract}
A new aspect of unification is presented in this paper. This aspect depends on two postulates of our recent theory concerning light propagation in a specific medium. The special theory of relativity is demonstrated as a reflection of our first postulate, regarding the existence of multiple, equivalent rest frames in our medium. The second postulate is concerned with energy forms in nature, and it deals with the quantum behavior of light and wave behavior of matter. By using this postulate, we are able to justify the existence of these two phenomena. As a consequence of this, gravity as described by general relativity is unified as a background-independent interaction under the same postulate.
\end{abstract}

\section{Keywords}

Sama Aether Theory, Theory of Relativity, Quantum Theory, Light, Matter Waves, Gravity, Rest Motion, String Theory, Discrete Space

\section{Introduction}

We have recently introduced a new medium for light propagation in the universe. The new theory is called Sama Aether Theory (SAT) [1]. Regarding aether (ether) as a medium for light propagation, there are two facts that should be mentioned. The first is that many physicists believe that light or electromagnetic (EM) waves do not need a medium to propagate from one place to another. The second is that the concept of light's medium has become experimentally problematic, since Michelson-Morely experiment [2]. Therefore, ether is considered as unnecessary addition to physics. We also believe that the quest for a medium for light propagation is not interesting any more. The major goal behind SAT is not to introduce a medium for light propagation in space-time, although it is spontaneously achieved in our theory. Our major goal is to merge the concepts of the theory of relativity and the quantum theory in one major idea, based only on two simple postulates. 


\section{The Special Theory of Relativity as a Reflection of SAT's First Postulate}

There are two aspects to Albert Einstein's relativity theory: the special theory of relativity (STR) and the general theory of relativity (GTR) [3]. We will leave GTR to the fourth section, because it is also linked to our second postulate, and here, in this section, we will consider only the STR.

In our previous paper [1], the first postulate of SAT states that the ether has multiple, equivalent rest frames. Any observer is said to be in rest frame, with respect to ether, when the velocity between him and the ether is classically equals to zero. Therefore, it represents an inertial state. Now, since the observer is existed in a rest frame, with respect to ether, any light signal will propagates with the same speed $(c)$ in all spatial directions, since the observer in a rest frame, as:

$$
\left|\boldsymbol{c}_{x}\right|=\left|\boldsymbol{c}_{y}\right|=\left|\boldsymbol{c}_{z}\right|=C
$$

As we have shown [1] the existence of multiple rest frames means that any observer can escape from one rest frame, and gets into another rest frame. As a result of this, the speed of light will be the same in all spatial directions in both frames, since they are rest frames. Also, since the rest frames are physically equivalent, this will mean that laws of physics will be the same in all rest frames. Therefore, a frame of reference in any rest frame of ether represents an inertial frame. From here, we can say that, the first postulate of SAT simply means two consequences: the laws of physics are the same in any inertial frame of reference, and the speed of light has the same value in any inertial frame of reference. These two consequences represent the idea behind the special theory of relativity.

\section{The Quantum Theory as a Reflection of SAT's Second Postulate}

What is light? Where do matter waves come from? To answer these questions, we have to make a quick review on our second postulate of SAT, which is the kinetic energy, and the potential energy are the only forms of energy for the ether's waves [1]. The kinetic energy and the potential energy represent mechanical forms of energy which means that, they are always carried on particles or quanta. These quanta have a linear momentum, and acquire energy from their motion. From the prospect of our theory, the EM wave represents a "field" wave that propagates in the ether. This wave is just a field wave, which means that it has only one velocity vector, which points toward the direction of wave propagation. This will mean that the quanta or particles which carry the wave's energy will have only one possible direction of movement, which is the direction of propagation. Therefore, light propagates as a wave, but when we try to detect its energy experimentally, it will be detected in discrete, localized spots. This is in comparison with the mechanical waves like sound wave which have two velocity vectors. The first is associated with the amplitude of the wave, and the second is the propagation velocity vector. From here, the particles which carry the energy of the sound waves will move according to the amplitude's velocity vector. This is by itself results in a "continuous" distribution of energy along the wave front.

The second postulate of our theory arises from the fact that the energy forms in nature are limited. The electric (magnetic) field represents a physical entity that spreads "continuously" in space-time. This field carries energy, but what type or form of energy? This is the important question that should be asked. The classical physicists simply assumed that the electric field has its own form of energy, which is called the electric field energy. This form of energy was assumed to spread continuously with the field itself. From here, the energy of the EM wave will spread continuously along the wave front.

This contradicts the experimental evidences which indicate that the energy of the EM wave is detected in discrete, localized spots. From here, we can simply say that, the EM field lacks its own form of energy, and it carries the kinetic energy instead. As a result of this, light is defined as a wave that carries kinetic energy. This also means that, there will be no need to assume that light has a dual nature. This is because, the wave theory by itself does not forbid a wave from carrying its energy in the kinetic form, just like saying sound wave is a wave that carries kinetic energy. Also, we were able to derive the formula $E=h v$ from simple classical calculations, and to predict the existence of Planck's constant from the same classical concepts.

The framework of our theory requires that the EM waves not to be the only waves in ether [1]. This means that the existence of another field waves in ether which shares the same medium with the EM waves, but with different amplitude field values. Our second postulate will be applied on these field waves, since they are wave in ether. The result of this will be just like that of EM waves. The energy of these different field waves will be carried on quanta. We have shown that the value of these different amplitudes can be visualized as " $\psi$ ", where 
$|\psi|^{2}$ is simply related to the probability of experimentally detecting the field's quantum in a specific point in space and time. Since $\psi$ is a general value for any field wave. This means that different field values with different physical properties will be detected as different particles or quanta with different physical properties [1]. The problem with concept is that, these waves "matter waves" carry rest energy $E_{0}$, which contributes to wave's energy , and it is neither kinetic nor potential energy, which violates our postulate. We were able to solve this problem by proving that the kinetic energy has three different forms in nature. The first is the classical form $K E_{1} \approx \frac{1}{2} m_{0} v^{2}$, and the second form is $K E_{2}=E=p c$ which represents the energy of a massless particle. The third form represents the rest energy itself, which we will consider it in the next section, when we speak about gravity. Finally, we assumed a non-mechanical structure of ether which means that it is not composed of particles (matter) and therefore energy (momentum) that can be directly detected in the laboratory. This is by itself explains why the ether does not appear or interfere with the sensitive experiments of modern physics, especially those of the EM phenomena, as if they were performed in a true vacuum, if the ether is really existed.

\section{Gravity and Unification of Physics}

In order to build a theory that unifies the physical interactions in one theoretical framework, we must overcome two major problems. The first is that the theory has to provide us with one picture or idea about the universe. In other words, describing the different physical interactions as different reflections of one origin. The second is to overcome the problem of the infinities that arises from the uncertainty principle.

String theory [4], overcomes these two problems spontaneously. First, it provides us with the idea of the string which represents the fundamental element in the universe. The basic idea behind this theory is that this string vibrates with different states of vibrations, and each state of vibration corresponds to a different physical particle.

From here, the unification of physical interactions becomes prominent, since we have one string, with different states of vibrations that give us different particles, which mediate the interactions that we observe in nature (Figure 1).

Furthermore, this string has a one dimensional extension in space-time, which means that the problem of infinities is solved, since it arises from a "zero" spatial distances of physical interactions.

The problem with this current approach for unification in the string theory is that it describes the gravitational interaction by a particle "graviton" which represents a state of the string itself. This represents a problem because it makes gravity a background-dependent interaction, which represents a major contradiction to the picture of gravity in the GTR. This is because GTR describes gravity a curvature in the fabric of space-time which makes gravity a background-independent interaction. From here, if we accept this approach of unification, we will have to undo the picture of gravity that is given by GTR.

In our ether theory SAT, we have a similar approach of unification. Here, we do not have strings, but we have the ether, and as we mentioned in the previous section, the ether has different waves with different values of amplitude. Each amplitude represents a specific field wave in the same ether ([1]; Figure 2).

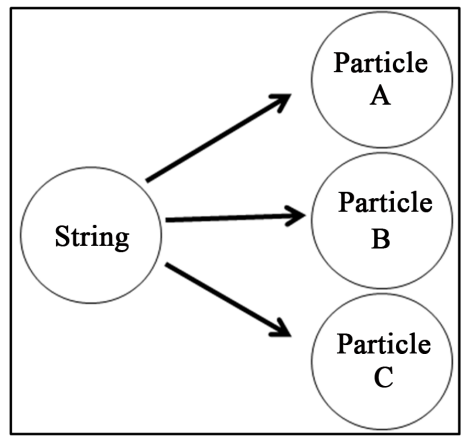

Figure 1. The basic idea in string theory is that, each state of vibration in the string corresponds to different particle, for example a hypothetical state of vibration (A) gives a hypothetical particle (A). A hypothetical state of vibration (B), gives a hypothetical particle (B), and so on. 


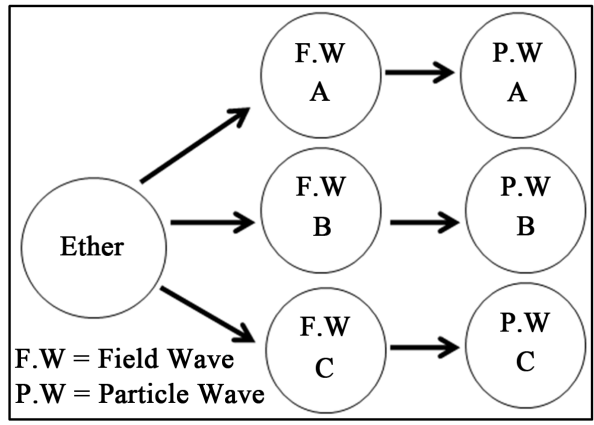

Figure 2. In SAT, The ether has different amplitudes, and each amplitude represents a specific field wave. The hypothetical field wave (A), and the hypothetical field wave (B), will be detected practically as waves of probabilities for particle (A), and particle (B) respectively.

A major difference, from the string theory is that we have used a background-independent approach to unify gravity. To explain this, in the previous section, we mentioned that from the second postulate of SAT, we have calculated three forms or types of kinetic energy. We defined two forms, and here, we define the third form of kinetic energy $K_{3}$. For a spherically symmetric body, with a massive rest energy $E_{0}$ :

$$
K E_{3}=\frac{r_{0} c^{2} v_{0}^{2}}{2 G}=E_{0}\left(v_{0}\right)
$$

where $c$ is the speed of light, $G$ is the universal gravitational constant, $r_{0}$ is radius of the body, and $v_{0}$ is a new term defined as the rest speed of the body [1]. From here, we can see that the magnitude of this rest energy $E_{0}$ depends on the speed $v_{0}$, which make it energy that is acquired from motion "kinetic energy".

Now, let us review the concept of this rest speed by taking three simple situations as an example. Initially, consider a classical particle that is located at the origin at the moment $\left(t_{1}\right)$. At the moment $\left(t_{2}\right)$, the particle had moved to the right side, and its current position became $(\boldsymbol{A})$. Since the initial position of the particle was the origin, the magnitude of the displacement $(\Delta x)$ is equal to the current position of the particle. It is extremely important to note that every position that was located to the right side at the moment $\left(t_{1}\right)$, had a value of $(\boldsymbol{x})$. But, at $\left(t_{2}\right)$ the value has changed to $\boldsymbol{x}-\Delta \boldsymbol{x}$. Now, since $\Delta \boldsymbol{x}$ is equal to $\boldsymbol{A}$. By substitution, this simply gives $\boldsymbol{x}-\boldsymbol{A}$ (Figure 3).

Our second situation is identical to the first, except that the particle has moved to the left side. We will see that every position that is located to the left side of the particle has changed from $-\boldsymbol{x}$ to $-\boldsymbol{x}-(-\boldsymbol{A})$, where $-\boldsymbol{A}$ is the position of the particle at $\left(t_{2}\right)$ (Figure 4 ).

Now, by look at our third situation in Figure 5, we should ask ourselves, where is the particle located at the moment $\left(t_{2}\right)$ ?

By looking at the right side of the particle, we will see that the value of every position has changed to $\boldsymbol{x}-\boldsymbol{A}$, which indicates that the current position of the particle is $\boldsymbol{A}$, since it is equal to the value of displacement. Also, by looking at the left side of the particle, we will say that the particle is located at $-\boldsymbol{A}$, since the value of every position has been changed to $-\boldsymbol{x}-(-\boldsymbol{A})$. From here, we will have to conclude that at the moment $\left(t_{2}\right)$, the particle is located in two positions, which are $\boldsymbol{A}$, and $-\boldsymbol{A}$, or by simple mathematical expression $\boldsymbol{A}+(-\boldsymbol{A})$. This is equal to a single value, which is zero. This means that the particle has not changed its position between $\left(t_{1}\right)$, and $\left(t_{2}\right)$. If we divide our previous expression over the time interval between these two moments, we will get $\boldsymbol{v}_{0}+\left(-\boldsymbol{v}_{0}\right)$, where $v_{0}$ represents the rest speed of the particle.

From here, we can say that the particle's velocity $\boldsymbol{v}$ is zero, but its rest velocity $\boldsymbol{v}_{0}$ is not zero. We call it rest speed, because it describes the particle's motion while it is in rest, without changing its position. Or in other words, the particle is moving into two opposite spatial direction simultaneously. The value of this rest speed $v_{0}$ determines how the values of the surrounding positions around the particle change with time, and these changes have the same magnitude, but with opposite direction (Figure 5).

The main conclusion from GTR is that it describes the gravitational field around a massive body (like Earth) as a curvature in the fabric of the space-time that results from its content of energy. We are familiar with the fact that both spatial and time measurements are distorted with motion for a stationary observer. Therefore, by using 


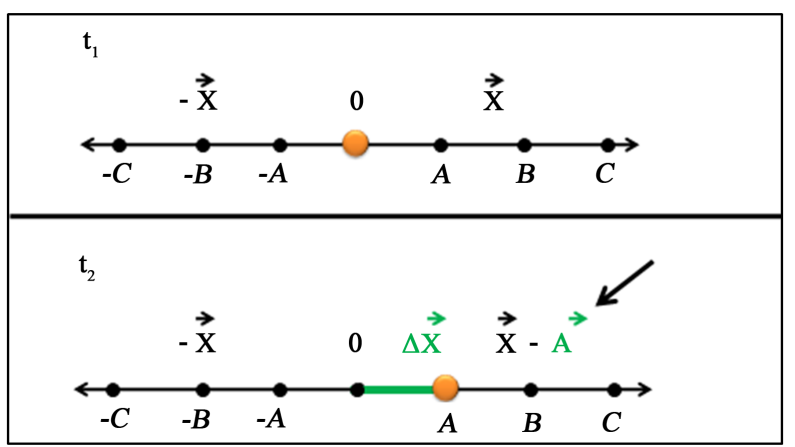

Figure3. $\boldsymbol{x}-\boldsymbol{A}$.

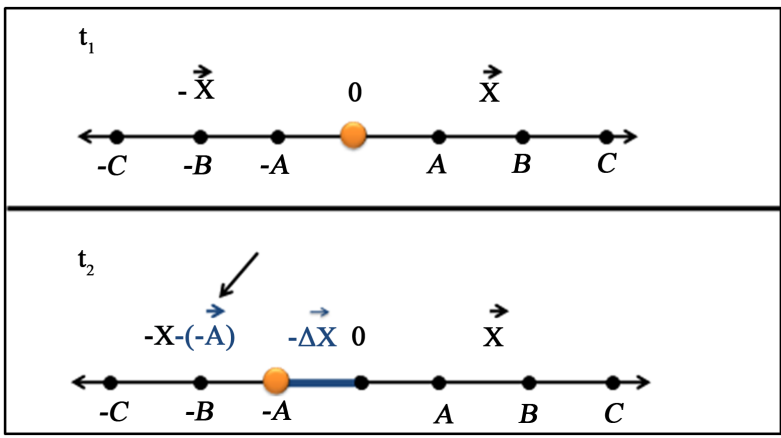

Figure 4. $-x$ to $-x-(-A)$.

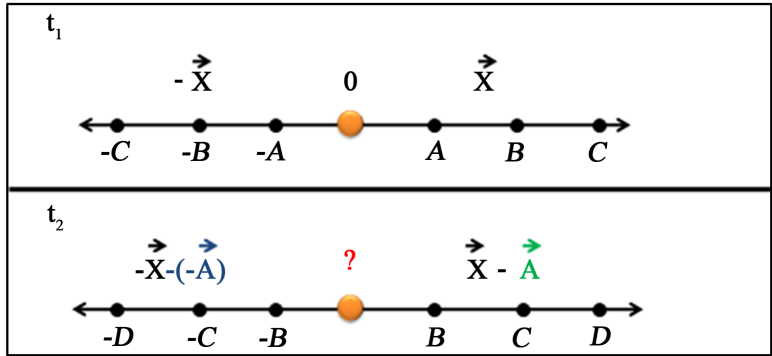

Figure 5. $-\boldsymbol{X}-(-\boldsymbol{A})$.

the notion of rest speed, and connecting it with the rest energy as a new form of kinetic energy, we were able to show that this physical effect is simply reflected as a curvature in the fabric of the space-time [1]. We were able to derive mathematical formulas that governed space-time curvature outside a static, symmetrically spherical mass. Our calculations were based on simple, elementary mathematics. This is in comparison with the more complicated tensor calculus of GTR. The results were identical to the Schwarzschild solution [1] [5] of GTR.

This is by itself illustrates a simple approach to unify gravity as background-independent interaction, since we used one principle, which is SAT's second postulate to predict the existence of gravity, and the quantum phenomena. The problem with our approach is that we used classical calculations that ignore the uncertainty principle, or in other words it ignores the "infinities" problem. The problem of infinities arises from the concepts of quantum mechanics and classical GTR. The uncertainty principle states that in order to observe a small region of space-time we need to concentrate a large amount of energy and momentum. However, GTR implies that if we concentrate too much energy and momentum in a small region, that region will collapse into a black hole and disappear. From here we can see that the problem of infinities simply disappear if the space is not infinitely divisible, or in other words, if we have an elementary spatial granule or quantum. Because at this situation, we will have a specific limit for our observation, which is the "granule" level. Therefore, we cannot go to infinite small spatial regions "spatial points" in our calculations that result in the appearance of the infinities because they do 
not exist. In a previous paper [6], we were able to show an evidence, for the existence of these elementary spatial granules, although we were unable to calculate its exact size or shape.

\section{Conclusion}

We have shown that the existence of multiple rest frames results in existence of equivalent inertial frames of reference, with a constant speed of light propagation in all frames, which represents the basic concept behind special relativity. We have also shown that the wave-particle duality does not exist in our theory. We only have the ether's wave, which shares the same medium, but with different amplitude values. As a result of this, the rest energy is defined as a form of kinetic energy. This unifies gravity as a background-independent interaction under the same principle.

\section{References}

[1] Isam, A. (2015) American Research journal of Physics, 1, 36-55.

[2] Michelson, A.A. and Morley, E.W. (1887) American Journal of Science, 34, 333-345. http://dx.doi.org/10.2475/ajs.s3-34.203.333

[3] Einstein, A. (1961) Relativity: The Special and the General Theory. Three Rivers Press, New York (Published 1995).

[4] Davies, P.C.W. and Brown, J., Eds. (1988) Superstrings: A Theory of Everything? Cambridge University Press, Cambridge.

[5] McMahon, D. (2006) Relativity Demystified. The McGraw-Hill Companies, New York, 203-229.

[6] Isam, A. (2013) Jordan Journal of Physics, 6, 73-77. 